EPJ Web of Conferences 53, 07008 (2013)

DOI: $10.1051 /$ epjconf/20135307008

(C) Owned by the authors, published by EDP Sciences, 2013

\title{
Recent results from LHCf
}

\author{
Gaku Mitsuka \\ Nagoya University
}

\begin{abstract}
The Large Hadron Collider forward experiment measured very forward neutral particle spectra in LHC proton-proton collisions in early 2010. In this paper we will discuss the energy and transverse momentum spectra of neutral pion at the $7 \mathrm{TeV}$ proton-proton collision, and the inclusive photon energy spectra taken at the $900 \mathrm{GeV}$ collisions. The spectra in both collision energies are also compared with the predictions of several hadronic interaction models that are often used for high energy particle physics and for modeling ultra high energy cosmic ray showers.
\end{abstract}

\section{INTRODUCTION}

The Large Hadron Collider forward (LHCf) experiment [1] has been designed to measure the hadronic production cross sections of neutral particles emitted in very forward angles in proton-proton collisions at the LHC, including zero degrees. The LHCf detectors have the capability for precise measurements of forward high-energy inclusive-particle-production cross sections of photons, neutrons, and possibly other neutral mesons and baryons. The analyses in this paper concentrate on obtaining (1) the inclusive production rate for $\pi^{0} \mathrm{~s}$ in the rapidity range larger than $y=8.9$ as a function of the $\pi^{0}$ transverse momentum, and (2) the inclusive production rate for photons in the rapidity ranges $\eta>10.15$ and $8.77<\eta<9.46$ as a function of the photon energy.

This work is motivated by an application to the understanding of Ultra-High-Energy Cosmic-Ray (UHECR) phenomena, which are sensitive to the details of soft $\pi^{0}$ production at extreme energy. It is known that the lack of knowledge about forward particle production in hadronic collisions hinders the interpretation of observations of UHECR [2,3]. Although UHECR observations have made notable advances in the last few years [4-10], critical parts of the analysis depend on Monte Carlo (MC) simulations of air shower development that are sensitive to the choice of the hadronic interaction model. It should also be remarked that the LHC has reached $7 \mathrm{TeV}$ collision energy, which in the laboratory frame of UHECR observations is equivalent to $2.6 \times 10^{16} \mathrm{eV}$, and tis energy is above the "knee" region of the primary cosmic ray energy spectrum $\left(\sim 4 \times 10^{15} \mathrm{eV}\right)[11]$. The data provided by LHCf should then provide a useful bench mark for the MC codes that are used for the simulation of UHECR atmospheric showers.

This paper is organized as follows. In Sec. 2 the LHCf detectors are described. Sec. 3 summarizes the conditions for taking data and Sec. 4 summarizes the MC simulation methodology. In Sec. 5 the analysis framework is described. The analyses results are then presented in Sec. 6 and Sec. 7. Finally, concluding remarks are found in Sec. 8.

\section{THE LHCF DETECTORS}

Two independent LHCf detectors, called Arm1 and Arm2, have been installed in the instrumentation slots of the target neutral absorbers (TANs) [12] located $\pm 140 \mathrm{~m}$ from the ATLAS interaction point

\footnotetext{
a e-mail: Gaku.Mitsuka@cern.ch
}

This is an Open Access article distributed under the terms of the Creative Commons Attribution License 2.0, which permits unrestricted use, distribution, and reproduction in any medium, provided the original work is properly cited. 

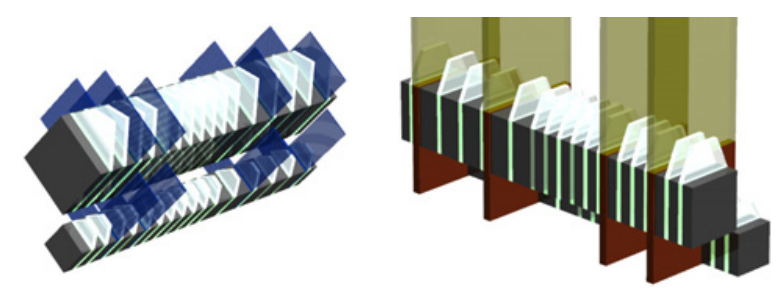

Figure 1. Schematic views of the Arm1 (left) and Arm2 (right) detectors. The transverse sizes of the calorimeters are $20 \times 20 \mathrm{~mm}^{2}$ and $40 \times 40 \mathrm{~mm}^{2}$ in Arm1, and $25 \times 25 \mathrm{~mm}^{2}$ and $32 \times 32 \mathrm{~mm}^{2}$ in Arm 2 .

(IP1) and at zero degree collision angle. Figure 1 shows schematic views of the Arm1 (left) and Arm2 (right) detectors. Inside a TAN the beam-vacuum-chamber makes a Y-shaped transition from a single common beam tube facing IP1 to two separate beam tubes joining to the arcs of the LHC. Charged particles produced at IP1 and directed towards the TAN are swept aside by the inner beam separation dipole magnet D1 before reaching the TAN. Consequently only neutral particles produced at IP1 enter the LHCf detector. At this location the LHCf detectors cover the pseudorapidity range from 8.7 to infinity for zero degree beam crossing angle. With a maximum beam crossing angle of $140 \mu \mathrm{rad}$, the pseudorapidity range can be extended to 8.4 to infinity.

Each LHCf detector has two sampling and imaging calorimeters composed of 44 radiation lengths $\left(X_{0}\right)$ of tungsten and 16 sampling layers of $3 \mathrm{~mm}$ thick plastic scintillator. The transverse sizes of the calorimeters are $20 \times 20 \mathrm{~mm}^{2}$ and $40 \times 40 \mathrm{~mm}^{2}$ in Arm1, and $25 \times 25 \mathrm{~mm}^{2}$ and $32 \times 32 \mathrm{~mm}^{2}$ in Arm2. The smaller and larger calorimeters are called as "small tower" and "large tower", respectively. The small towers cover zero degree collision angle. Four X-Y layers of position sensitive detectors are interleaved with the layers of tungsten and scintillator in order to provide the transverse positions of the showers. Scintillating fiber ( $\mathrm{SciFi}$ ) belts are used for the Arm1 position sensitive layers and silicon micro-strip sensors are used for Arm2. Readout pitches are $1 \mathrm{~mm}$ and $0.16 \mathrm{~mm}$ for Arm1 and Arm2, respectively.

More detail on the scientific goals and the construction and performance of the detectors can be found in previous reports [13-17].

\section{SUMMARY OF THE CONDITIONS FOR TAKING DATA}

\section{1 proton-proton collisions at $\sqrt{s}=7 \mathrm{TeV}$}

The experimental data used for the $\pi^{0}$ analysis of this paper were obtained on May 15th and 16th 2010 during proton-proton collisions at $\sqrt{s}=7 \mathrm{TeV}$ with zero degree beam crossing angle (LHC Fill 1104). Data taking was carried out in two different runs: the first run was on May 15th from 17:45 to 21:23, and the second run was on May 16th from 00:47 to 14:05. The events that were recorded during a luminosity optimization scan and a calibration run were removed from the data set for this analysis.

The range of total luminosity of the three crossing bunch pairs was $\mathcal{L}=(6.3-6.5) \times 10^{28} \mathrm{~cm}^{-2} \mathrm{~s}^{-1}$ for the first run and $\mathcal{L}=(4.8-5.9) \times 10^{28} \mathrm{~cm}^{-2} \mathrm{~s}^{-1}$ for the second run. These ranges of luminosity were ideal for the LHCf data acquisition system. The integrated luminosities for the data analysis reported in this paper were derived from the counting rate of the LHCf Front Counters [18], and were $2.53 \mathrm{nb}^{-1}$ (Arm1) and $1.90 \mathrm{nb}^{-1}$ (Arm2) after taking the live time percentages into account. The average live time percentages for the first/second run were $85.7 \% / 81.1 \%$ for Arm 1 and $67.0 \% / 59.7 \%$ for Arm2. The live time percentages for the second run were smaller than the first run owing to a difference in the trigger schemes. In both runs the trigger efficiency achieved was $>99 \%$ for photons with energy $E>100 \mathrm{GeV}[19]$. 


\section{UHECR 2012}

The events containing more than one collision in a single bunch crossing (pile-up events) could potentially cause a bias in the $p_{\mathrm{T}}$ spectra. For example combinatorial single-hits from different collisions within a single bunch crossing might be identified as multi-hit events from a single collision and removed from the analysis. (Multi-hit events have two showers in a single calorimeter and are eliminated from the data analysis. The production rates are later corrected for this cut.) However it can be shown that pile-up events are negligible for the LHCf data taking conditions of this report. Given that a collision has occurred, the probability of pile-up $\left(P_{\text {pileup }}\right)$ is calculated from the Poisson probability distribution for $n$ collisions $P_{\text {poi }}(n)$ according to $P_{\text {pileup }}=P_{\text {poi }}(n \geq 2) / P_{\text {poi }}(n \geq 1)$. With the highest bunch luminosity $\mathcal{L}=2.3 \times 10^{28} \mathrm{~cm}^{-2} \mathrm{~s}^{-1}$ used in this analysis, an inelastic cross section $\sigma_{\text {inel }}=73.6 \mathrm{mb}$ and the revolution frequency of LHC $f_{\text {rev }}=11.2 \mathrm{kHz}$, the pile-up probability is $P_{\text {pileup }} \sim 0.07$. However considering that the acceptance of the LHCf calorimeter for inelastic collisions is $\sim 0.03$, only $0.2 \%$ of events have more than one shower event in a single calorimeter due to pile-up and this is negligible.

Detailed discussions of pile-up effects and background events from collisions between the beam and residual gas molecules in the beam tube can be found in previous reports $[1,19]$.

\section{2 proton-proton collisions at $\sqrt{s}=900 \mathrm{GeV}$}

The data sets used in the photon analysis were taken on 2, 3 and 27 May 2010 during the LHC operations with proton-proton collisions at $\sqrt{s}=900 \mathrm{GeV}$, which correspond to the LHC fill identification numbers (Fill ID) 1068, 1069 and 1128, respectively. In these fills, the LHC operated with one crossing bunch and one non-crossing bunch at IP1 in Fill IDs 1068 and 1069 and with four crossing bunches and three non-crossing bunches at IP1 in Fill ID 1128. The luminosity $\mathcal{L}$ at IP1 during these fills was measured by the ATLAS experiment [20]. The luminosity during Fill ID 1068 and 1069 were $\mathcal{L}=8--3 \times 10^{27} \mathrm{~cm}^{-2} \mathrm{~s}^{-1}$ and $\mathcal{L}=12--4 \times 10^{27} \mathrm{~cm}^{-2} \mathrm{~s}^{-1}$, respectively. The total luminosity of the four crossing bunches in Fill ID 1128 was approximately $\mathcal{L}=8 \times 10^{27} \mathrm{~cm}^{-2} \mathrm{~s}^{-1}$. The total integrated luminosity $\int \mathcal{L} d t$ during the LHCf operations in the three fills was $0.30 \mathrm{nb}^{-1}$. The uncertainty of the luminosity determination is $\pm 21 \%$ [20]. The inelastic cross-section $\sigma_{\text {inel }}$ for a $\sqrt{s}=900 \mathrm{GeV}$ proton-proton collision was estimated to be $\sigma_{\text {inel }}=53.0 \mathrm{mb}$ from the predictions of the total crosssection and the elastic cross section, which are based on the recent experimental results [21, 22]. The number of inelastic collisions $N_{\text {inel }}$ during the three fills was calculated to be $1.58 \times 10^{7}$.

During the $\sqrt{s}=900 \mathrm{GeV}$ fills, the LHCf operations were performed with a high-gain operation of the PMTs for the sampling layers to detect photons with energies as low as $50 \mathrm{GeV}$ with a nearly $100 \%$ trigger efficiency and with a lower threshold with respect to the $\sqrt{s}=7 \mathrm{TeV}$ data. The typical PMT gain for the high-gain operations was $3-5$ times higher than the nominal gain that was used to obtain the $7 \mathrm{TeV}$ data. Neither the saturation of PMTs nor the range of ADC caused problems because the maximum energy of the incident photons were expected to be 8 times lower than those for the $7 \mathrm{TeV}$ data. The average DAQ live times during the LHCf $\sqrt{s}=900 \mathrm{GeV}$ operations was $99.2 \%$ (Arm1) and 98.0\% (Arm2). Because of the very low luminosity and the low event rate per inelastic collision, the probability of the pile-up of events was $<10^{-4}$, negligibly small.

\section{SUMMARY OF THE METHODOLOGY FOR PERFORMING MONTE CARLO SIMULATIONS}

MC simulation consists of three steps: (1) proton-proton interaction event generation at IP1, (2) transport from IP1 to the LHCf detectors and (3) the response of the LHCf detectors.

Proton-proton interaction events at $\sqrt{s}=7 \mathrm{TeV}$ and $\sqrt{s}=900 \mathrm{GeV}$ and the resulting flux of secondary particles and their kinematics are simulated with Cosmos (version 8.81). Cosmos acts as the front end for the external hadronic interaction models (QGSJET II-03 [23], DPMJET 3.04 [24], SIBYLL 2.1 [25] and EPOS 1.99 [26]) that describe the proton-proton interactions. While for PYTHIA8.145 [27, 28], proton-proton interaction events are simulated by PYTHIA 8.145 standalone. 
Next, the generated secondary particles are transported in the beam pipe from IP1 to the TAN, taking account of the deflection of charged particles by the Q1 quadrupole and D1 beam separation dipole, particle decay, and particle interaction with the beam pipe and the Y-shaped beam-vacuumchamber transition made of copper ( $1 X_{0}$ projected thickness in front of the LHCf detectors). Charged particles are swept away by the D1 magnet before reaching the LHCf detectors. This simulation uses the EPICs library [29] (version 7.49) and a part of COSMOS. EPICS deals with the transport of secondary particles. Particle interactions with the residual gas molecules inside the beam pipe are not simulated. Contamination from beam-gas background events in the data set used for analysis is estimated to be only $\sim 0.1 \%$ and has no significant impact on the $p_{\mathrm{T}}$ and energy spectra reported.

Finally the simulations of the showers produced in the LHCf detectors and their response are carried out for the particles arriving at the TAN using the COSMOS and EPICs libraries. The survey data for detector position and random fluctuations equivalent to electrical noise are taken into account in this step. The Landau-Pomeranchuk-Migdal effect $[30,31]$ that longitudinally lengthens an electromagnetic shower at high energy is also considered. A change of the $p_{\mathrm{T}}$ and energy spectra caused by LPM effects is only at the $1 \%$ level since the reconstruction of energy deposited in the calorimeters is carried out to a sufficiently deep layer where the energy of electromagnetic shower is almost perfectly deposited within the calorimeter.

The simulations of the LHCf detectors are tuned to test beam data taken at the CERN SPS in 2007 [15]. The validity of the detector simulation was checked by comparing the shower development and deposited energy for each calorimeter layer to the results obtained by the FLUKA library [32].

\section{ANALYSIS FRAMEWORK}

\subsection{Multi-hit selection}

As noted later, an energy reconstruction relies on the sum of the visible-energy-deposits on each scintillator layer. Hence, in the case of multiple hits on one tower (multi-hit), the energy of each entering particle can not be reconstructed individually, then such misreconstruction possibly causes the incorrect energy spectrum. Therefore the multi-hit selection is crucial in the data analysis in LHCf. The multi-hit selection is based on the peak-finding algorithm that has been implemented in the TSpectrum [33] class in ROOT [34].

According to the estimation with the MC simulations, single-hit events can be collected with the efficiency larger than $98 \%$ and its energy dependence is less than $2 \%$. Also the efficiency has no significant dependence on the PID criteria.

\subsection{Reconstruction of shower position}

Determination of the incidence position of entering particles on the detector is one of the most important reconstruction processes for two reasons:

- Because of the small size of calorimeter, a part of particle shower is unavoidably leaked out from the calorimeter. Shower leakage can be compensated by adding the corresponding energy deposit which can vary as a function of incidence position.

- Invariant mass of two photons decayed from $\pi^{0}$ can be reconstructed by the energy on each tower and opening angle. Opening angle is geometrically determined in terms of the incidence positions on each tower and the distance between the decay point of $\pi^{0}$ and the detector.

Energy deposit along the lateral direction is fitted by Eq. (1), and the fitted variable $b$ is the reconstructed incidence position.

$$
f(a, b, c) \propto c \frac{a}{\left[(x-b)^{2}+a\right]^{3 / 2}} .
$$


Best-fit values of $a, b$ and $c$ are extracted by minimizing the $\chi^{2}$ function (i.e., a goodness-of-fit test) using the MINOS-MINUIT algorithm [35]. The estimated resolution using the toy MC simulations and test beam data for a single photon with energy $E>100 \mathrm{GeV}$ is better than $200 \mu \mathrm{m}$ and $100 \mu \mathrm{m}$ for Arm 1 and Arm2, respectively [16, 36].

\subsection{Shower leakage correction}

A part of shower developed in the detector is unavoidably leaked out from the calorimeter because of its small lateral size (in the case of tungsten, the Moliere radius is about $9 \mathrm{~mm}$ ). The fraction of leakage can be geometrically estimated by the MC simulation for each incidence position. Events with the particles hitting on the outer $2 \mathrm{~mm}$ from the calorimeter edge are not used in the analysis because of the difficulty to compensate the large amount of shower leakage.

Another aspect of the shower leakage is that leaked to the other side of tower (i.e. leakage-in) which has the impact in the reconstruction of $\pi^{0}$ mass, in which the sensitivity and resolution rely on the precise reconstruction of the energy of the pair of two photons.

\subsection{Energy reconstruction}

Shower energy is reconstructed using the visible-energy-deposit on the calorimeter. Thanks to the sufficiently dense absorber made by tungsten (totally $44 X_{0}$ ), the longitudinal development of electromagnetic shower can be perfectly detected, while that of hadronic shower can be partially leaked out from the last layer of the scintillators.

Sum of the energy deposits (called as SumdE) in units of MIP is defined as

$$
S u m d E=\sum_{i=2}^{11} E_{d e p}^{i}+2 \sum_{i=12}^{13} E_{d e p}^{i}
$$

where index $i$ denotes the $i$-th scintillator. Coefficient 2 in the second term corresponds to the twice longer tungsten-absorber ( $4 X_{0}$ for each layer) layers than that in the former layers ( $2 X_{0}$ for each layer). The energy deposit in the 1st layer is disregarded in the energy reconstruction since this layer is highly influenced by the background events induced by collisions between beam and residual gas. Conversion constants from SumdE to reconstructed energy are determined by the MC simulation from the energy threshold up to $3.5 \mathrm{TeV}$.

\subsection{Particle identification}

Particle identification (PID) is one of the important processes executed in the data analysis, especially both purity and efficiency must be addressed. If only keeping higher purity is focused, the efficiency may fall down because of the severe cut and vice versa.

Among many proposed approaches, the $L_{90 \%}$ method is employed in the current data analysis. This method is based on the shower development along the longitudinal direction, where an electromagnetic shower may develop faster than a hadronic shower.

$L_{90 \%}$ is defined as the shower depth in units of $X_{0}$ where an integral of energy deposits in the calorimeter from $0 X_{0}$ to $L_{90 \%}$ achieves $90 \%$ of the total energy deposit as described in Eq. (3).

$$
\int_{0}^{L_{90 \%}} E_{d e p}=0.9 \int_{0}^{44 X_{0}} E_{d e p} .
$$



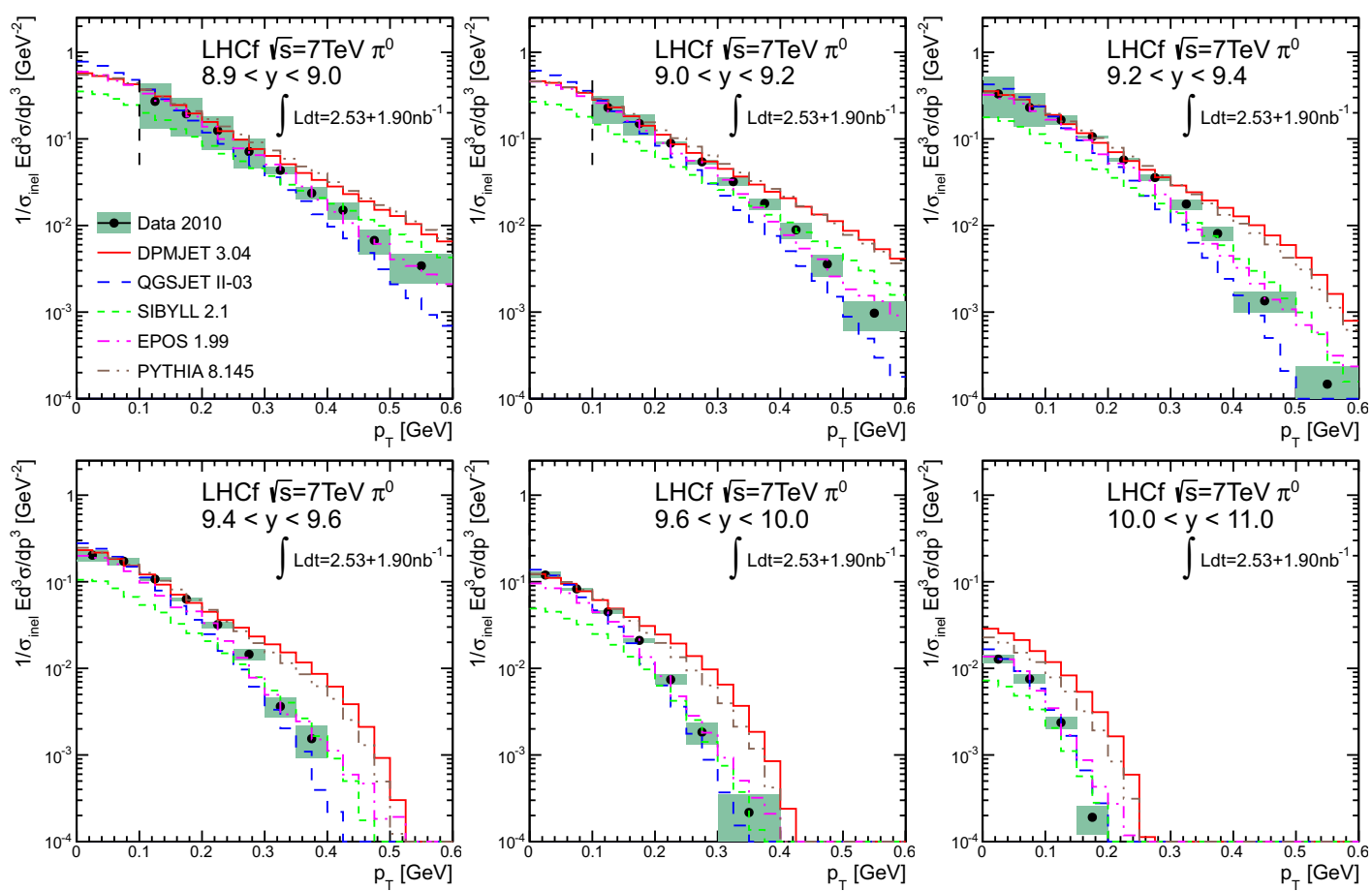

Figure 2. Combined $p_{\mathrm{T}}$ spectra of the Arm 1 and Arm 2 detectors (black dots) and the total uncertainties (shaded rectangles) compared with the predicted spectra by hadronic interaction models.

\section{RESULTS OF $\pi^{0}$ ANALYSIS AT $\sqrt{S}=7$ TEV}

The combined $p_{\mathrm{T}}$ spectra of the Arm1 and Arm2 detectors are presented in Fig. 2 for six ranges of rapidity $y$ : 8.9 to $9.0,9.0$ to $9.2,9.2$ to $9.4,9.4$ to $9.6,9.6$ to 10.0 and 10.0 to 11.0. The spectra in Fig. 2 are after all corrections discussed in previous sections have been applied. The inclusive production rate of neutral pions is given by the expression

$$
\frac{1}{\sigma} E_{\text {inel }} \frac{d^{3} \sigma}{d p^{3}}=\frac{1}{N_{\text {inel }}} \frac{d^{2} N\left(p_{\mathrm{T}}, y\right)}{2 \pi \cdot p_{\mathrm{T}} \cdot d p_{\mathrm{T}} \cdot d y} .
$$

$\sigma_{\text {inel }}$ is the inelastic cross section for proton-proton collisions at $\sqrt{s}=7 \mathrm{TeV} . E d^{3} \sigma / d p^{3}$ is the inclusive cross section of $\pi^{0}$ production. The number of inelastic collisions, $N_{\text {inel }}$, used for normalizing the production rates of Fig. 2 has been calculated from $N_{\text {inel }}=\sigma_{\text {inel }} \int \mathcal{L} d t$, assuming the inelastic cross section $\sigma_{\text {inel }}=73.6 \mathrm{mb}$. This value for $\sigma_{\text {inel }}$ has been derived from the best COMPETE fits [22] and the TOTEM result for the elastic scattering cross section [21]. Using the integrated luminosities reported in Sec. $3.1, N_{\text {inel }}$ is $1.85 \times 10^{8}$ for Arm 1 and $1.40 \times 10^{8}$ for Arm2. $d^{2} N\left(p_{\mathrm{T}}, y\right)$ is the number of $\pi^{0} \mathrm{~s}$ detected in the transverse momentum interval $\left(d p_{\mathrm{T}}\right)$ and the rapidity interval $(d y)$ with all corrections applied. In Fig. 2, the $68 \%$ confidence intervals incorporating the statistical and systematic uncertainties are indicated by the shaded green rectangles.

For comparison, the $p_{\mathrm{T}}$ spectra predicted by various hadronic interaction models are also shown in Fig. 2. The hadronic interaction models that have been used in Fig. 2 are DPMJET 3.04 (solid, red), QGSJET II-03 (dashed, blue), SIBYLL 2.1 (dotted, green), EPOS 1.99 (dashed dotted, magenta), and PYTHIA 8.145 (default parameter set, dashed double-dotted, brown). In these MC simulations, $\pi^{0} \mathrm{~s}$ from short lived particles that decay within $1 \mathrm{~m}$ from IP1, for example $\eta \rightarrow 3 \pi^{0}$, are also counted to be consistent with 


\section{UHECR 2012}
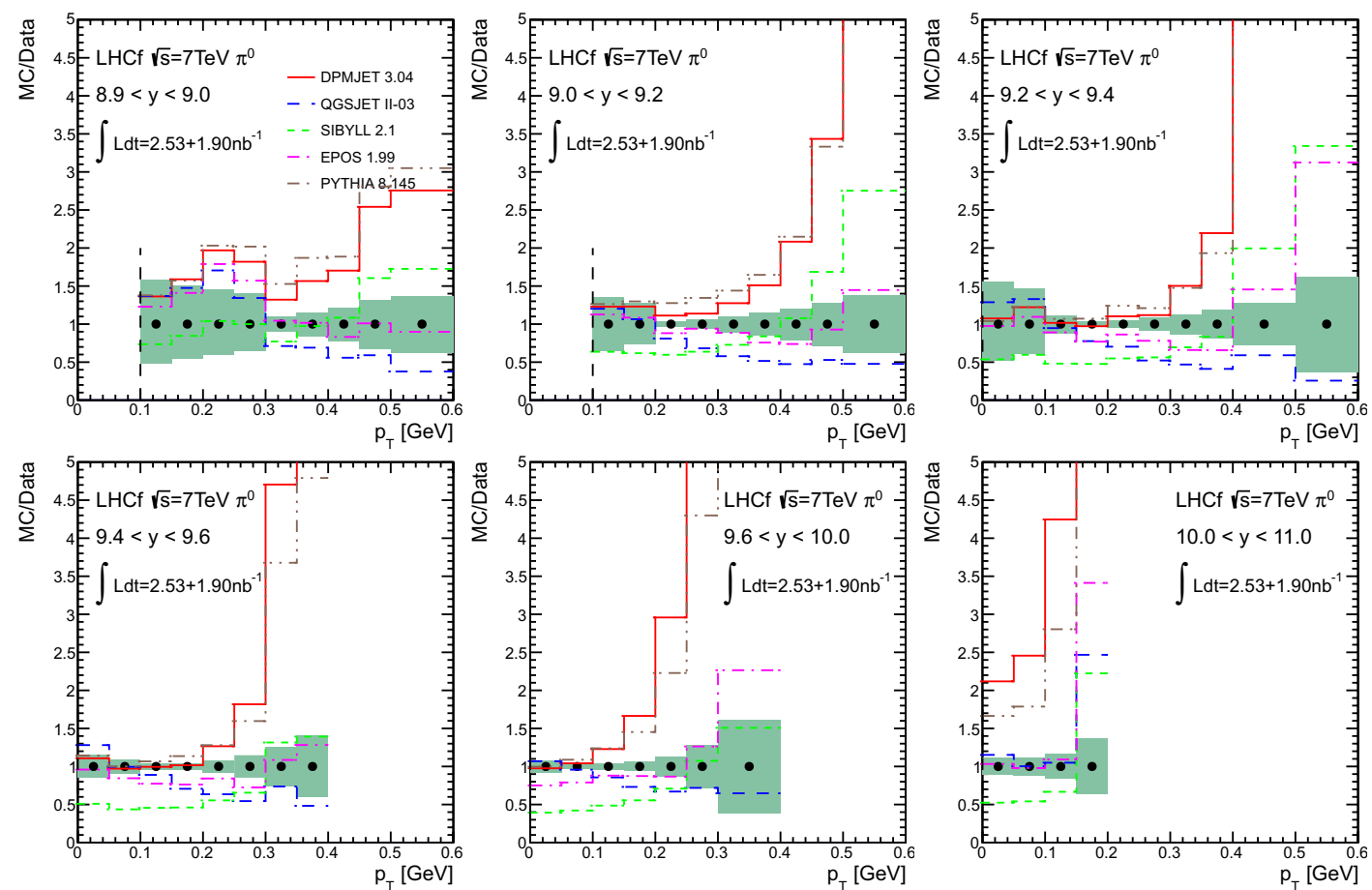

Figure 3. Ratio of the combined $p_{\mathrm{T}}$ spectra of the Arm 1 and Arm 2 detectors to the predicted $p_{\mathrm{T}}$ spectra by hadronic interaction models. Shaded areas indicate the range of total uncertainties of the combined $p_{\mathrm{T}}$ spectra.

the treatment of the experimental data. Note that, since the experimental $p_{\mathrm{T}}$ spectra have been corrected for the influences of the detector responses, event selection efficiencies and geometrical acceptance efficiencies, the $p_{\mathrm{T}}$ spectra of the interaction models may be compared directly to the experimental spectra as presented in Fig. 2.

Figure 3 presents the ratios of $p_{\mathrm{T}}$ spectra predicted by the various hadronic interaction models to the combined $p_{\mathrm{T}}$ spectra. Error bars have been taken from the statistical and systematic uncertainties. A slight step found around $p_{\mathrm{T}}=0.3 \mathrm{GeV}$ in $8.9<y<9.0$ is due to low $p_{\mathrm{T}}$ cutoff of the Arm1 data.

Several points can be made about Fig. 3. First, DPMJET 3.04 and PYTHIA 8.145 show overall agreement with the LHCf data for $9.2<y<9.6$ and $p_{\mathrm{T}}<0.2 \mathrm{GeV}$, while the expected $\pi^{0}$ productions rates by both models exceed the LHCf data as $p_{\mathrm{T}}$ becomes large. The latter observation can be explained by the baryon/meson production mechanism that has been employed in both models. More specifically, the "popcorn model" $[37,38]$ is used to produce baryons and mesons through string breaking, and this mechanism tends to lead to hard pion spectra. SIBYLL 2.1, which is also based on the popcorn model, also predicts harder pion spectra than the experimental data, although the expected $\pi^{0}$ yield is generally small.

On the other hand, QGSJET II-03 predicts $\pi^{0}$ spectra that are softer than the LHCf data and the other models. This might be due to the fact that only one quark exchange is allowed in the QGSJET model. The remnants produced in a proton-proton collision are likewise baryons with relatively small mass, so fewer pions with large energy are produced.

Among hadronic interaction models tested in this analysis, EPOS 1.99 shows the best overall agreement with the LHCf data. However EPOS 1.99 behaves softer than the data in the low $p_{\mathrm{T}}$ region, $p_{\mathrm{T}} \lesssim 0.4 \mathrm{GeV}$ in $9.0<y<9.4$ and $p_{\mathrm{T}} \lesssim 0.3 \mathrm{GeV}$ in $9.4<y<9.6$, and behaves harder in the large $p_{\mathrm{T}}$ 

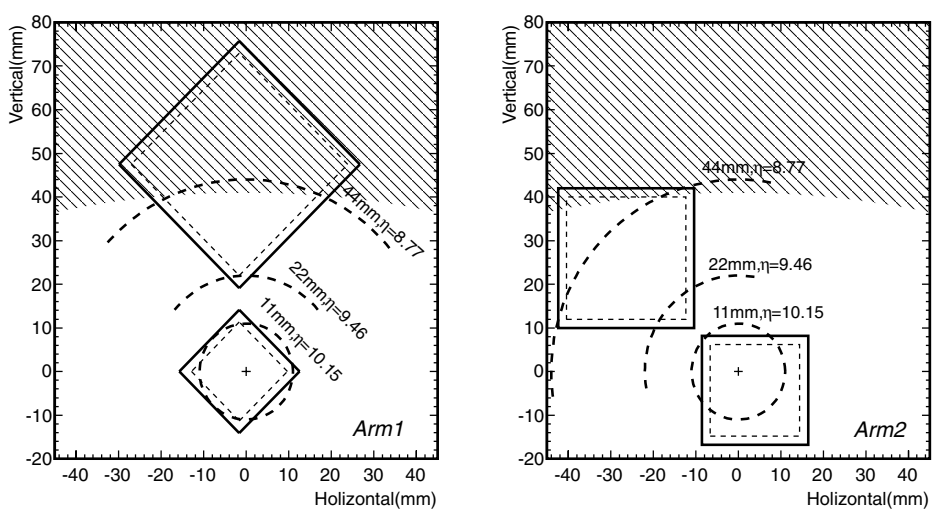

Figure 4. The cross-sections of the calorimeters viewed from IP1, left for Arm1 and right for Arm2. The cross marks on the small calorimeters indicate the projections of the zero-degree collision angle onto the detectors ("beam center"). The shaded areas in the upper parts of the figure indicate the shadows of the beam pipes located between IP1 and the detectors, where the detectors are insensitive to the detection of IP1 proton-proton collision products. The dashed squares indicate the border of a $2 \mathrm{~mm}$ edge cut described in Sect. 5.3. The circles and the arcs indicate the distance $(r)$ from the beam center of $11 \mathrm{~mm}, 22 \mathrm{~mm}$ and $44 \mathrm{~mm}$, and the pseudorapidity $(\eta)$ of 10.159 .46 and 8.77 , respectively. In this analysis, the events in the regions of $r<11 \mathrm{~mm}$ and $22 \mathrm{~mm}<r<44 \mathrm{~mm}$ we are used.

region. Specifically a dip found in the ratio of EPOS 1.99 to the LHCf data for $y>9.0$ can be attributed to the transition between two pion production mechanisms: string fragmentation via cut Pomeron process (low energy $\sim$ low $p_{\mathrm{T}}$ for the fixed rapidity) and remnants of projectile/target (high energy $\sim$ large $p_{\mathrm{T}}$ for the fixed rapidity) [39].

\section{RESULTS OF PHOTON ANALYSIS AT $\sqrt{S}=900 \mathrm{GEV}$}

To reduce a possible pseudorapidity $\eta$ dependence when comparing and combining the energy spectra measured by the two Arms, we selected Arm2 events with a pseudorapidity range similar to that of Arm1. For the small tower, we selected events with the distance $r$ from the beam center less than $11 \mathrm{~mm}$, which corresponded to the pseudorapidity range of $\eta>10.15$ (the circles in Fig. 4). Similarly, for the large tower, we set the conditions as $22 \mathrm{~mm}<r<44 \mathrm{~mm}$, which corresponded to the pseudorapidity range of $8.77<\eta<9.46$ (the arcs in Fig. 4). The calorimeters did not uniformly cover the pseudorapidity ranges as shown in Fig. 4 . We confirmed that there was a negligible pseudorapidity dependence of the energy spectra inside each pseudorapidity range. The reconstructed photon energy spectra of Arm1 and Arm2 are shown in Fig. 5 in units of differential cross-sections $d \sigma / d E d \Omega$, where $E$ is the photon energy and $\Omega$ is the solid angle. The differential cross-section was calculated as $d \sigma / d E d \Omega=1 /\left(\int L d t\right) d N / d E d(\cos \theta) d \phi$, where $N$ is the number of events in each energy bin, $\int \mathcal{L} d t$ is the integrated luminosity after the correction for the DAQ live time for each Arm and $\theta$ and $\phi$ are the polar and the azimuthal angles with respect to the beam axis, respectively. Considering the geometrical acceptance of the calorimeters, the averages of the polar angle $\langle\theta\rangle$ are $39 \mu \mathrm{rad}$ and $234 \mu \mathrm{rad}$ for the small and the large tower, respectively. The error bars in Fig. 5 indicate the statistical uncertainty; the hatched areas show the systematic errors in which the particle identification and the beam position uncertainties were taken into account. Because the systematic uncertainties due to the energy determination may be correlated between Arm1 and Arm2 [19], they were not taken into account in Fig. 5. The two spectra from Arm 1 and Arm 2 in each pseudorapidity region gave consistent results within the statistical and the systematic errors.

The combined energy spectra of Arm1 and Arm2 are shown in Fig. 6 as weighted averages, with the weights taken to be the square of the inverse of the errors in each energy bin. The error bars 

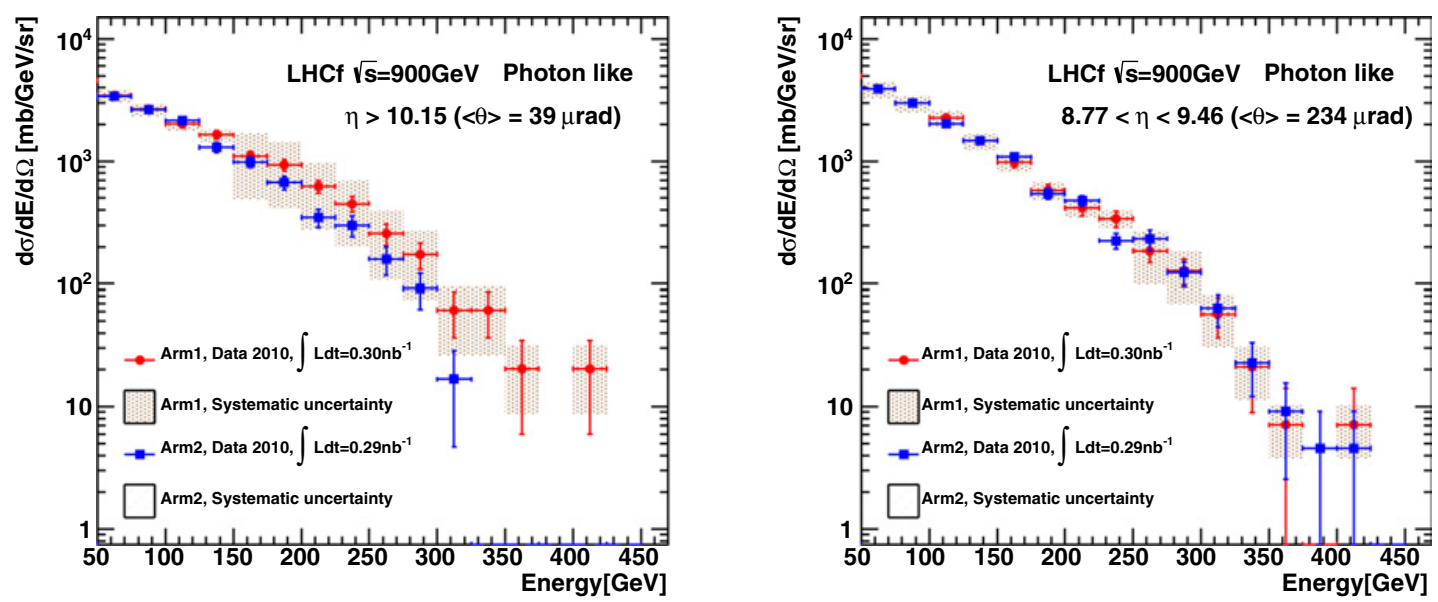

Figure 5. Photon energy spectra measured by Arm1 (red circles) and Arm2 (blue rectangles) in units of differential cross-section $d \sigma / d E d \Omega$. The left and the right panels show the results of the small towers and the large towers, respectively. The pseudorapidity coverages of the small and the large towers are $\eta>10.15$ and $8.77<\eta<9.46$, respectively. Considering the geometrical shape of the calorimeters, the averages of the polar angle $\langle\theta\rangle$ with respect to the beam axis are $39 \mu \mathrm{rad}$ and $234 \mu \mathrm{rad}$ for the small and the large towers, respectively. The error bars indicate the statistical errors, and the hatched areas indicate the total systematic uncertainties that come from particle identification and the "beam center" position.
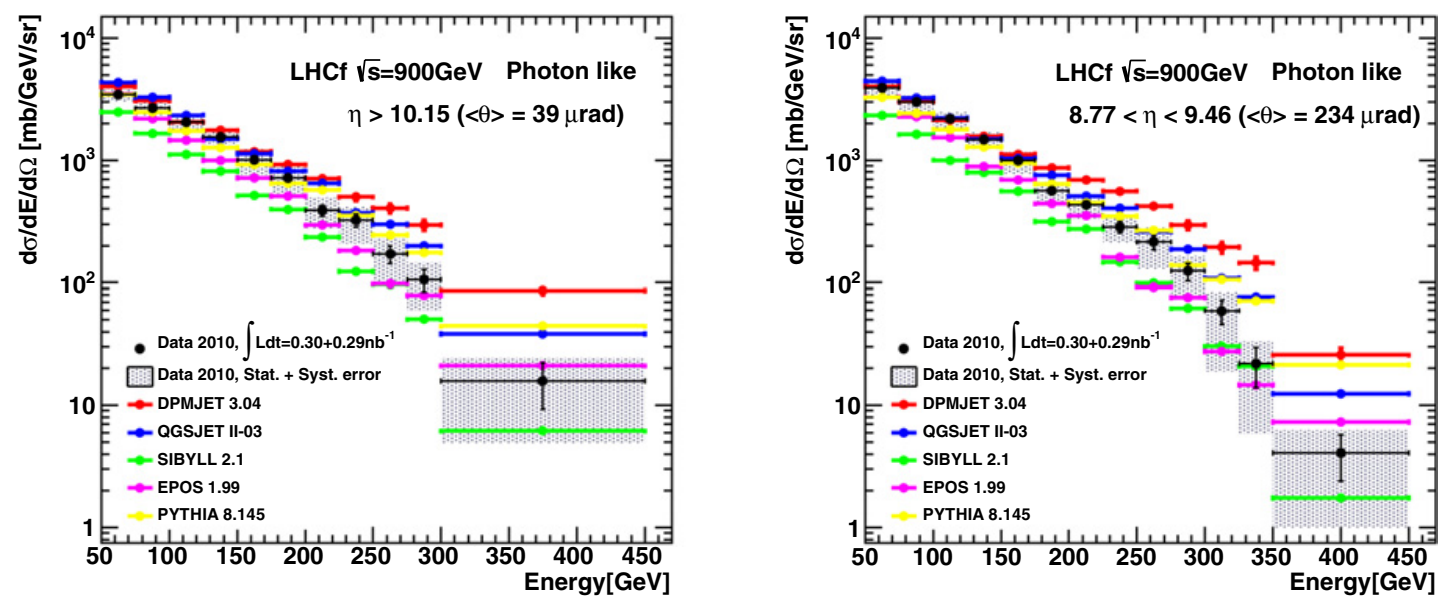

Figure 6. Combined Arm 1 and Arm 2 photon energy spectra compared with MC predictions. The data from Arm1 and Arm 2 correspond to the integral luminosities of 0.30 and $0.29 \mathrm{nb}^{-1}$, respectively. The left and the right panels are the results of the small $(\eta>10.15)$ and the large $(8.77<\eta<9.46)$ towers, respectively. The black points indicate the experimental data with the statistical uncertainty (error bars) and the total uncertainty, quadratical summation of the statistical and the systematic errors (black hatches). The systematic uncertainty of the luminosity determination $( \pm 21 \%)$ is not taken into account in the errors. The colored points indicate the results of MC predictions, QGSJET II-03 (blue), PYTHIA 8.145 (yellow), SIBYLL 2.1 (green), EPOS 1.99 (magenta) and DPMJET 3.04 (red). Only the statistical uncertainty of DPMJET 3.04 is shown by the error bars as representative of the models. 

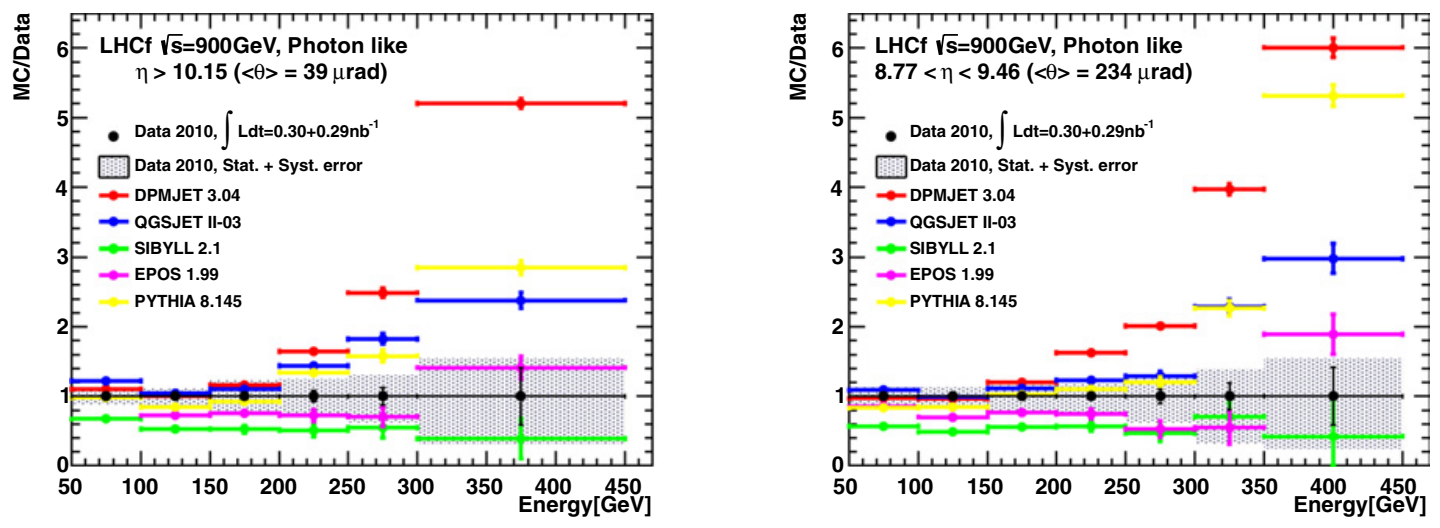

Figure 7. Ratio of the MC spectra divided by the data in each energy bin. The left and the right panels show the spectra for the pseudorapidity ranges of $\eta>10.15$ and $8.77<\eta<9.46$, respectively. The colored plots indicate the results of MC, QGSJET II-03 (blue), PYTHIA 8.145 (yellow), SIBYLL 2.1 (green), EPOS 1.99 (magenta) and DPMJET 3.04 (red). To describe the size of the errors, the experimental data are also shown on the ratio of unity with the statistical uncertainty (error bars) and the total uncertainty of data (the black hatches). The luminosity uncertainty was not included. The statistical uncertainties of each MC are shown as the error bars of MC.

of the data (black points) represent the statistical error; the hatches in the spectra represent the total uncertainty (quadratical summation of the statistical and the systematic errors). The sources of the systematic error are the particle identification and the beam position uncertainties. The energy scale errors were also included, assuming a correlation between the two Arms. Note that the uncertainty of the luminosity determination $( \pm 21 \%)$ is not shown in Fig. 6. It can introduce a constant vertical shift of the spectra, but it cannot change the shapes of the spectra. We see a smooth spectrum from each of the two pseudorapidity regions, considering the errors. The similarity of the two spectra suggests only a small pseudorapidity dependence between the two pseudorapidity regions.

In Figure 6, the predictions of the hadronic interaction models, QGSJET II-03, PYTHIA 8.145, SIBYLL 2.1, EPOS 1.99 and DPMJET 3.04, are also shown. The same analysis processes were applied to the MC simulations as to the experimental data except for the particle identification using $L_{90 \%}$ and its correction. For the analysis of the MC simulations, the known particle type was used. For better visibility, only the statistical errors for DPMJET 3.04 (red points) are shown by the error bars. Figure 7 shows the ratios of the MC spectra divided by the data in each energy bin. In Fig. 7, the statistical error of each MC is shown as the error bar of each point. The trends of the experimental data compared to each MC are similar for the two pseudorapidity ranges. EPOS 1.99 and SIBYLL 2.1 show a reasonable agreement with the spectral shape of the experimental data, whereas they predict lower cross-sections than the data. The other models, DPMJET 3.04, QGSJET II-03 and PYTHIA 8.145, are in good agreement with the data below $300 \mathrm{GeV}$ but predict harder energy spectra than the data above $300 \mathrm{GeV}$. The trends of the experimental data compared to the MC predictions in Fig. 7 are similar to those for the single-photon energy spectra in the pseudorapidity $\eta>10.94$ previously reported for $\sqrt{s}=7 \mathrm{TeV}$ proton-proton collisions [19].

\section{CONCLUSIONS}

The inclusive production of neutral pions in the rapidity range larger than $y=8.9$ at $\sqrt{s}=7 \mathrm{TeV}$ proton-proton collisions and the forward inclusive photon energy spectra in the pseudorapidity ranges of $\eta>10.15$ and $8.77<\eta<9.46$ for $\sqrt{s}=900 \mathrm{GeV}$ proton-proton collisions have been measured by the LHCf experiment in proton-proton collisions at the LHC in early 2010. Transverse momentum 


\section{UHECR 2012}

spectra of neutral pions and energy spectra of photons have been measured by two independent LHCf detectors, Arm1 and Arm2, and give consistent results.

The combined Arm1 and Arm2 spectra have been compared with the predictions of five hadronic interaction models, DPMJET 3.04, EPOS 1.99, PYTHIA 8.145, QGSJET II-03 and SIBYLL 2.1. For the neutral pion spectra, DPMJET 3.04, EPOS 1.99 and PYTHIA 8.145 agree with the LHCf combined results in general for the rapidity range $9.0<y<9.6$ and $p_{\mathrm{T}}<0.2 \mathrm{GeV}$. QGSJET II-03 has poor agreement with LHCf data for $8.9<y<9.4$, while it agrees with LHCf data for $y>9.4$. Among the hadronic interaction models tested in this paper, EPOS 1.99 shows the best overall agreement with the LHCf data even for $y>9.6$.

For the photon spectra, EPOS 1.99 and SIBYLL 2.1 reproduce well the shape of the experimental energy spectra, but they predict a lower cross section than the LHCf data. The other models predict harder spectra than the LHCf data above $300 \mathrm{GeV}$. These results of comparison exhibited features similar to those for the previously reported data for $\sqrt{s}=7 \mathrm{TeV}$ collisions.

\section{References}

[1] LHCf Technical Design Report, CERN-LHCC-2006-004

[2] R. Ulrich, R. Engel and M. Unger, Phys. Rev. D 83, 054026 (2011)

[3] R.D. Parsonsa, C. Bleveb, S.S. Ostapchenkoc, J. Knappa, Astroparticle Physics 34, 832-839 (2011)

[4] J. Abraham, et al., Phys. Rev. Lett. 101061101 (2008)

[5] P. Abreu, et al., Astropart. Phys. 34, 314-326 (2010)

[6] J. Abraham, et al., Phys. Rev. Lett. 104, 091101 (2010)

[7] R. Abbasi, et al., Astropart. Phys. 32, 53-60 (2009)

[8] R. U. Abbasi, et al., The Astrophysical Journal Letters 713, 64-68 (2010)

[9] R. U. Abbasi, et al., Phys. Rev. Lett. 104, 161101 (2010)

[10] H. Sagawa, AIPC 1367, 17-22, (2011)

[11] J. R. Hörandel, Astropart. Phys. 19, 193-220 (2003)

[12] W. C. Turner, E. H. Hoyer and N. V. Mokhov, Proc. of EPAC98, Stockholm, 368 (1998). LBNL Rept. LBNL-41964 (1998)

[13] O. Adriani, et al., JINST 3, S08006 (2008)

[14] T. Sako, et al., NIM A578, 146 (2007)

[15] T. Mase, et al., NIM A671, 129 (2012)

[16] O. Adriani, et al., JINST 5, P01012

[17] H. Menjo, et al., Astropart. Phys. 34, 513-520 (2011)

[18] K. Taki, et al., JINST 7 T01003 (2012)

[19] O. Adriani, et al., Phys. Lett. B 703, 128-134 (2011)

[20] The ATLAS collaboration, ATLAS-CONF-2010-060

[21] G. Antchev, et al. (The TOTEM Collaboration), EPL 9621002 (2011)

[22] K. Nakamura, et al.. (Particle Data Group), J. Phys. G 37, 075021 (2010)

[23] S. Ostapchenko, Nucl. Phys. Proc. Suppl. 151, 143 (2006)

[24] F. W. Bopp, J. Ranft, R. Engel and S. Roesler, Phys. Rev. C 77, 014904 (2008)

[25] E.-J. Ahn, R. Engel, T. K. Gaisser, P.Lipari and T. Stanev, Phys. Rev. D 80, 094003 (2009)

[26] K. Werner, F.-M. Liu and T. Pierog, Phys. Rev. C 74, 044902 (2006)

[27] T. Sjöstand, S. Mrenna and P. Skands, JHEP05, 026 (2006)

[28] T. Sjöstand, S. Mrenna and P. Skands, Comput. Phys. Comm. 178, 852 (2008)

[29] K. Kasahara, Proc. of 24th Int. Cosmic Ray. Conf. Rome 1, 399 (1995). EPICS web page, http://cosmos.n.kanagawa-u.ac.jp/

[30] L. D. Landau and I. J. Pomeranchuk, Dokl. Akad. Nauk 92, 535-536 (1965)

[31] A. B. Migdal, Phys. Rev. 103, 1811 (1956) 
[32] G. Battistoni, S. Muraro, P.R. Sala, F. Cerutti, A. Ferrari, S. Roesler, A. Fasso“, J. Ranft, AIP Conference Proceeding 896, 31-49, (2007). A. Ferrari, P.R. Sala, A. Fasso`, and J. Ranft, CERN2005-10 (2005), INFN/TC_05/11, SLAC-R-773

[33] M. Morhac et al., NIM A 401, 113-132 (1997), M. Morhac et al., NIM A 401, 385-408 (1997), M. Morhac et al., NIM A 443, 108-125 (2000)

[34] Rene Brun and Fons Rademakers, ROOT - An Object Oriented Data Analysis Framework, Proceedings AIHENP'96 Workshop, Lausanne, Sep. 1996, NIM 389, 81-86 (1997). See also http: //root.cern.ch/

[35] F. James and M. Roos, Computer Physics Communications 10, 343-367 (1975)

[36] M. Mizuishi et al., J. Phys. Soc. Jpn. 78 (2009) Suppl. A, pp. 173-176

[37] B. Andersson, G. Gustafson and T. Sjöstand, Phys. Scripta 32, 574 (1985)

[38] P. Edén and G. Gustafson, Z. Phys. C 75 41-49 (1997)

[39] T. Pierog, Private communication 